\title{
Vacuum stability and spontaneous violation of the lepton number at a low-energy scale in a model for light sterile neutrinos
}

\author{
João Paulo Pinheiro® and C. A. de S. Pires® \\ Departamento de Física, Universidade Federal da Paraíba, \\ Caixa Postal 5008, 58051-970 João Pessoa, Paraíba, Brasil
}

(Received 20 March 2020; accepted 2 July 2020; published 16 July 2020)

\begin{abstract}
It is well known that the standard model of electroweak interactions rests on a metastable vacuum. This can be fixed only by means of new physics. Presently neutrino physics provides the most intriguing framework to formulate new physics. This is so because, in addition to the problem with lightness for the active standard neutrinos, current MiniBooNE experimental results may indicate that sterile neutrinos exist and are light, too. In this case, it is reasonable to expect that the framework that yields light active and sterile neutrinos could stabilize the vacuum, too. To achieve this goal, we consider an extension of the standard model which involves new fermions in the form of right-handed neutrinos $\left(\nu_{R}\right)$ and new scalars in the form of triplets $(\Delta)$ and singlets $(\sigma)$. Within this framework, tiny masses are obtained when we consider that lepton number is spontaneously broken at low-energy scale, which means that $\Delta$ and $\sigma$ both develop very small vacuum expectation values. We investigate whether this setting leads to a stable vacuum. For this, we obtain the whole set of conditions over the quartic terms of the potential that ensures that the model is bounded from below and evaluate the renormalization group equation of the self-coupling of the Higgs. We show that in such a scenario the quartic coupling $\kappa$ of the interaction $\left(\Phi^{T} \Delta \Phi \sigma+\right.$ H.c. $)$, where $\Phi$ is the standard Higgs doublet, is responsible for the stability of the electroweak vacuum up to Planck scale. We also extract constraints over the parameters of the potential by means of lepton flavor violating processes and from invisible decay of the standardlike Higgs.
\end{abstract}

DOI: 10.1103/PhysRevD.102.015015

\section{INTRODUCTION}

Although researchers have devoted considerable attention to the study of extensions of the standard Higgs sector [1-3], relative little attention has been given to a Higgs sector involving triplets $(\Delta)$, doublets $(\Phi)$, and singlets $(\sigma)$ of scalars [4-6]. From now on, we shall refer to this case as the 3-2-1 model. This model is interesting in its own right. However, it becomes even more interesting when righthanded neutrinos are introduced, too. This is so because in this case the 3-2-1 model yields the most general neutrino mass matrix involving Majorana and Dirac mass terms for both neutrinos. Hence, when we assume that the lepton number is spontaneously violated at low-energy scale, right-handed neutrinos acquire light masses and may explain the recent MiniBooNE experimental results [7] by means of neutrino oscillation.

In this work, we derive the complete set of conditions that guarantee the potential of the 3-2-1 model to be

Published by the American Physical Society under the terms of the Creative Commons Attribution 4.0 International license. Further distribution of this work must maintain attribution to the author(s) and the published article's title, journal citation, and DOI. Funded by SCOAP ${ }^{3}$. bounded from below. For the specific case in which lepton number is spontaneously broken at low-energy scale, we obtain the spectrum of scalars of the model and discuss the stability of the vacuum by evaluating the renormalization group equation (RGE) of the self-coupling of the standardlike Higgs up to Planck scales. This case is particularly interesting because it encompasses a Majoron and a light $C P$-even scalar in their spectrum of scalars. We discuss the contributions of these scalars for the invisible decay channels of the standardlike Higgs and of the neutral gauge boson $Z$. We also obtain the constraints that lepton flavor violation (LFV) puts over the parameters of the potential. Regarding neutrino physics, we provide a solution, i.e., a set of values for the Yukawa couplings, that recovers the standard neutrino sector and provides at least one righthanded neutrino with mass resting on the $\mathrm{eV}$ scale and robustly mixed with the standard neutrinos in such a way that it accommodates current MiniBooNE results by means of neutrino oscillation and is in agreement with cosmological data.

This work is organized as follows. In Sec. II, we develop the main aspect of the model including neutrino masses, while in Sec. III we develop the scalar sector. In Sec. IV, we discuss the stability of the vacuum. In Sec. V, we present our final remarks. 


\section{THE 3-2-1 MODEL}

The leptonic sector of the model is composed of the standard content plus right-handed neutrinos in the singlet form,

$$
L_{i}=\left(\begin{array}{c}
\nu_{i} \\
\ell_{i}
\end{array}\right)_{L} ; \quad \ell_{i_{R}} ; \quad \nu_{i_{R}}
$$

where $i=e, \mu, \tau$.

The scalar sector is composed of the standard Higgs doublet, $\Phi$, one Higgs triplet, $\Delta$, and one Higgs singlet, $\sigma$, presented below,

$$
\Delta=\left(\begin{array}{cc}
\Delta^{0} & \frac{\Delta^{+}}{\sqrt{2}} \\
\frac{\Delta^{+}}{\sqrt{2}} & \Delta^{++}
\end{array}\right) ; \quad \Phi=\left(\begin{array}{c}
\phi^{0} \\
\phi^{-}
\end{array}\right) ; \quad \sigma .
$$

The quark sector is the standard one.

The most general potential involving this scalar content that conserves lepton number is composed of the following terms:

$$
\begin{aligned}
V(\sigma, \Phi, \Delta)= & \mu_{1}^{2} \sigma^{*} \sigma+\mu_{2}^{2} \Phi^{\dagger} \Phi+\mu_{3}^{2} \operatorname{tr}\left(\Delta^{\dagger} \Delta\right) \\
& +\lambda_{1}\left(\Phi^{\dagger} \Phi\right)^{2}+\lambda_{2}\left[\operatorname{tr}\left(\Delta^{\dagger} \Delta\right)\right]^{2}+\lambda_{3} \Phi^{\dagger} \Phi \operatorname{tr}\left(\Delta^{\dagger} \Delta\right) \\
& +\lambda_{4} \operatorname{tr}\left(\Delta^{\dagger} \Delta \Delta^{\dagger} \Delta\right)+\lambda_{5}\left(\Phi^{\dagger} \Delta^{\dagger} \Delta \Phi\right) \\
& +\beta_{1}\left(\sigma^{*} \sigma\right)^{2}+\beta_{2} \Phi^{\dagger} \Phi \sigma^{*} \sigma+\beta_{3} \operatorname{tr}\left(\Delta^{\dagger} \Delta\right) \sigma^{*} \sigma \\
& -\kappa\left(\Phi^{T} \Delta \Phi \sigma+\text { H.c. }\right) .
\end{aligned}
$$

With such lepton and scalar content, the Yukawa interactions that generate mass for all neutrinos of the model is given by

$\mathcal{L}_{Y}^{\nu}=\frac{1}{2} Y_{i j}^{L} L_{i}^{T} \Delta L_{j}+Y_{i j}^{D} \overline{L_{i}} \tilde{\phi} \nu_{R j}+\frac{1}{2} Y_{i j}^{R} \overline{\nu_{R i}^{C}} \nu_{R j} \sigma+$ H.c.

The Yukawa interactions of the charged fermions are the standard ones.

When the neutral scalars of the model develop vacuum expectation values (VEVs) other than zero, i.e., $\langle\sigma\rangle=\frac{v_{1}}{\sqrt{2}}$, $\langle\phi\rangle=\frac{v_{2}}{\sqrt{2}}$, and $\langle\Delta\rangle=\frac{v_{3}}{\sqrt{2}}$, the Yukawa interactions in Eq. (4) provide the following mass terms for the neutrinos:

$\mathcal{L}_{\text {mass }}^{D+M}=\frac{1}{2} \bar{\nu}_{L}^{C} M_{L} \nu_{L}+\bar{\nu}_{L} M_{D} \nu_{R}+\frac{1}{2} \bar{\nu}_{R}^{C} M_{R} \nu_{R}+$ H.c.,

with $\nu_{L}=\left(\nu_{e_{L}} \nu_{\mu_{L}} \nu_{\tau_{L}}\right)^{T}$ and $\nu_{R}=\left(\nu_{e_{R}} \nu_{\mu_{R}} \nu_{\tau_{R}}\right)^{T}$.

Considering the basis $\nu=\left(\nu_{L} \nu_{R}^{C}\right)^{T}$, we can simplify Eq. (5) to

$$
\mathcal{L}_{\text {mass }}^{D+M}=\frac{1}{2} \bar{\nu}^{C} M^{D+M} \nu+\text { H.c. },
$$

and the $6 \times 6$ symmetric mass matrix is given by

$$
M^{D+M}=\left(\begin{array}{cc}
M_{L} & M_{D}^{T} \\
M_{D} & M_{R}
\end{array}\right)
$$

where $M_{L}=Y^{L}\langle\Delta\rangle, M_{D}=Y^{D}\langle\phi\rangle$, and $M_{R}=Y^{R}\langle\sigma\rangle$. $M^{D+M}$ is the most general neutrino mass matrix. It involves Dirac and Majorana mass terms for both left- and righthanded neutrinos. The 3-2-1 model is the simplest model that generates this mass matrix in the case of spontaneous violation of the lepton number.

The relation between the flavor basis, $\nu$, and the physical ones, $N=\left(N_{1} N_{2} N_{3} N_{4} N_{5} N_{6}\right)^{T}$, is given by $N=U \nu$, with $U$ being the unitary matrix that diagonalizes $M^{D+M}$,

$$
U^{T} M^{D+M} U=M=\operatorname{diag}\left(M_{1} M_{2}\right),
$$

where $M_{1}=\operatorname{diag}\left(m_{1} m_{2} m_{3}\right)^{T}$ and $M_{2}=\operatorname{diag}\left(m_{4} m_{5} m_{6}\right)^{T}$.

To go further, we need to obtain information about the VEVs $v_{1}, v_{2}$, and $v_{3}$. For this, we have to develop the scalar sector of the model.

First, we expand the neutral scalar fields around their respective VEVs,

$$
\begin{aligned}
\sigma & =\frac{v_{1}}{\sqrt{2}}+\frac{R_{1}+I_{1}}{\sqrt{2}}, \\
\phi^{0} & =\frac{v_{2}}{\sqrt{2}}+\frac{R_{2}+I_{2}}{\sqrt{2}}, \\
\Delta^{0} & =\frac{v_{3}}{\sqrt{2}}+\frac{R_{3}+I_{3}}{\sqrt{2}},
\end{aligned}
$$

and obtain the set of minimum conditions required by the potential above to allow spontaneous breaking of the symmetries of the model which include the global $B-L$ symmetry,

$$
\begin{aligned}
v_{1}\left(\mu_{1}^{2}+\beta_{1} v_{1}^{2}+\frac{1}{2} \beta_{2} v_{2}^{2}+\frac{1}{2} \beta_{3} v_{3}^{2}\right)-\frac{1}{2} \kappa v_{2}^{2} v_{3} & =0, \\
v_{2}\left(\mu_{2}^{2}+\lambda_{1} v_{2}^{2}+\frac{1}{2} \lambda_{3} v_{3}^{2}+\frac{1}{2} \lambda_{5} v_{3}^{2}+\frac{1}{2} \beta_{2} v_{1}^{2}-\kappa v_{1} v_{3}\right) & =0, \\
v_{3}\left(\mu_{3}^{2}+\lambda_{2} v_{3}^{2}+\frac{1}{2} \lambda_{3} v_{2}^{2}+\lambda_{4} v_{3}^{2}+\frac{1}{2} \lambda_{5} v_{2}^{2}+\frac{1}{2} \beta_{3} v_{1}^{2}\right)-\frac{\kappa v_{1} v_{2}^{2}}{2} & =0 .
\end{aligned}
$$

Upon analyzing this set of constraints, observe that the first and third relations yield

$$
v_{1} \approx \frac{\mu_{3}}{\mu_{1}} v_{3} .
$$

The parameters $\mu_{1}, \mu_{3}$, and $v_{1}$ are free to take any value, while $v_{3}$ is constrained by the rho parameter. This is so because $v_{3}$ gives different contributions to the mass of the standard gauge bosons $W^{ \pm}$and $Z^{0}$ modifying, in this way, the standard expression of the Veltman $\rho$ parameter [8] to 
$\frac{1+2\left(v_{3} / v_{2}\right)^{2}}{1+4\left(v_{3} / v_{2}\right)^{2}}$. As consequence of the fact that the experimental value of the rho parameter is near unity, we have the constraint $\frac{v_{3}}{v_{2}} \ll 1$. Then the direct proportionality among $v_{1}$ and $v_{3}$ provided by Eq. (11) hints that $v_{1}<v_{2}$, which implies that right-handed neutrinos may be light particles, too. That is very nice.

A strong reason for the existence of light right-handed neutrinos is the explanation of short-baseline neutrino results (LSND and MiniBooNE) [7,9] by means of neutrino oscillation. In this case, the adequate value for $v_{1}$ is one that accommodates at least one right-handed neutrino with mass around $\mathrm{eV}$ with robust mixing with the standard neutrinos and that is in conciliation with cosmology. We follow this line of reasoning here.

The current scenario of neutrino physics involving MiniBooNE and LSND experimental results may be accommodated within our model with the following set of values for the VEVs,

$v_{1}=10^{5} \mathrm{eV} ; \quad v_{2}=246 \mathrm{GeV} ; \quad v_{3}=1 \mathrm{eV}$,

and the following set of values for the Yukawa couplings,

$Y_{D}=\left(\begin{array}{ccc}3,6 \times 10^{-13} & 5,74 \times 10^{-13} & 5,74 \times 10^{-14} \\ -2,21 \times 10^{-13} & -3,45 \times 10^{-10} & 5,75 \times 10^{-13} \\ -7,07 \times 10^{-13} & 5,75 \times 10^{-12} & 5,75 \times 10^{-13}\end{array}\right)$,

$Y_{L}=\left(\begin{array}{ccc}6,20 \times 10^{-3} & -4,11 \times 10^{-3} & -1,25 \times 10^{-2} \\ -4,11 \times 10^{-3} & 3,90 \times 10^{-1} & 1,95 \times 10^{-2} \\ -1,25 \times 10^{-2} & 1,95 \times 10^{-2} & 3,83 \times 10^{-2}\end{array}\right)$,

$Y_{R}=\left(\begin{array}{ccc}1,40 \times 10^{-5} & 4,75 \times 10^{-12} & 4,52 \times 10^{-12} \\ 4,75 \times 10^{-12} & 10^{-1} & 5,08 \times 10^{-15} \\ 4,52 \times 10^{-12} & 5,08 \times 10^{-15} & 10^{-1}\end{array}\right)$

On substituting all of these values into $M^{D+M}$, given in Eq. (7), we have that its diagonalization provides $m_{1}=2 \times 10^{-4} \mathrm{eV} ; \quad m_{2}=8,6 \times 10^{-3} \mathrm{eV} ; \quad m_{3}=5 \times 10^{-2} \mathrm{eV}$,

$m_{4}=1,4 \mathrm{eV} ; \quad m_{5}=10^{4} \mathrm{eV} ; \quad m_{6}=10^{4} \mathrm{eV}$.

The mixing matrix, $U$, responsible for the diagonalization of $M^{D+M}$ and relating the basis $\nu$ to $N$, as in Eq. (8), is given by

$$
U=\left(\begin{array}{cccccc}
0,83 & 0,54 & -0,12 & 0,045 & 10^{-5} & 10^{-6} \\
-0,25 & 0,59 & 0,72 & -0,03 & -6 \times 10^{-3} & 10^{-5} \\
0,44 & -0,6 & 0,69 & -0,09 & 10^{-4} & 10^{-5} \\
-0,045 & 0,03 & 0,09 & 1 & \sim 0 & \sim 0 \\
10^{-6} & 10^{-4} & 10^{-4} & \sim 0 & 1 & \sim 0 \\
10^{-6} & 10^{-5} & 10^{-4} & \sim 0 & \sim 0 & 1
\end{array}\right) .
$$

The values of $m_{1}, m_{2}$, and $m_{3}$ given in Eq. (16) and the upper left $3 \times 3$ submatrix of $U$ accommodate the current solar and atmospheric neutrino oscillation data. A nice thing to observe is that the mixing angles among $N_{4}, \nu_{\mu}$, and $\nu_{e}$, together with the mass value of $m_{4}$, are in such a way that they allow the explanation of neutrino anomalies suggested by the data from short-baseline (SBL) neutrino experiments by means of neutrino oscillation. Finally, observe in $U$ that $N_{5}$ and $N_{6}$ practically decouple from the other neutrinos. In other words, this case recovers the $3+1$ sterile neutrino scenario.

A problem with models involving an $\mathrm{eV}$ sterile neutrino is that they create tension with current cosmological data [10]. We discuss this point later.

\section{SCALAR SECTOR}

We saw in the previous section that the scenario we are developing here is capable of accommodating neutrino physics including SBL anomalies such as LSND and MiniBooNE. This provides a strong reason for us to go deep into the development of such a case. Thus, in this section we perform a careful analysis of the spectrum of scalars of the model that is in consonance with the previous section, which means that we should consider the VEVs respecting the hierarchy $v_{3} \ll v_{1} \ll v_{2}$.

\section{A. Spectrum of scalars}

We now start developing the $C P$-even sector. Considering the basis $\left(R_{1} R_{3} R_{2}\right)$, the potential above together with the minimum conditions provides

$$
M_{R}^{2}=\left(\begin{array}{ccc}
2 \beta_{1} v_{1}^{2}+\frac{1}{2} \kappa v_{2}^{2} \frac{v_{3}}{v_{1}} & \beta_{3} v_{1} v_{3}-\frac{1}{2} \kappa v_{2}^{2} & \beta_{2} v_{1} v_{2}-\kappa v_{2} v_{3} \\
\beta_{3} v_{1} v_{3}-\frac{1}{2} \kappa v_{2}^{2} & 2\left(\lambda_{2}+\lambda_{4}\right) v_{3}^{2}+\frac{1}{2} \kappa v_{2}^{2} \frac{v_{1}}{v_{3}} & \left(\lambda_{5}+\lambda_{3}\right) v_{2} v_{3}-\kappa v_{1} v_{2} \\
\beta_{2} v_{3} v_{2}-\kappa v_{2} v_{1} & \left(\lambda_{5}+\lambda_{3}\right) v_{2} v_{3}-\kappa v_{1} v_{2} & 2 \lambda_{1} v_{2}^{2}
\end{array}\right)
$$


The complexity of this mass matrix prevents us from achieving a closed analytic form of the eigenvalues/eigenvectors. In this case, the best that we can do is a simplification based on our assumptions regarding the parameter space. In this way, according to the hierarchy of the VEVs that we have assumed here, this matrix may be approximated by

$$
M_{R}^{2} \approx\left(\begin{array}{ccc}
\frac{1}{2} \kappa v_{2}^{2} \frac{v_{3}}{v_{1}} & -\frac{1}{2} \kappa v_{2}^{2} & \sim 0 \\
-\frac{1}{2} \kappa v_{2}^{2} & \frac{1}{2} \kappa v_{2}^{2} \frac{v_{1}}{v_{3}} & \sim 0 \\
\sim 0 & \sim 0 & 2 \lambda_{1} v_{2}^{2}
\end{array}\right) .
$$

This means that $R_{2}$ decouples from the other ones, while $R_{1}$ and $R_{3}$ mix among themselves to form $H_{1}$ and $H_{3}$ according to the following relation:

$$
\left(\begin{array}{c}
H_{1} \\
H_{3}
\end{array}\right)=U_{R}\left(\begin{array}{l}
R_{1} \\
R_{3}
\end{array}\right) ; \quad R_{2}=H_{2},
$$

where

$$
U_{R} \approx\left(\begin{array}{cc}
1 & \epsilon \\
-\epsilon & 1
\end{array}\right) ; \quad \epsilon \approx \frac{v_{3}}{v_{1}}
$$

The masses are given by

$m_{H_{1}}^{2} \approx \frac{2 \beta_{2}^{2} v_{1}^{2}}{\kappa}, \quad m_{H_{3}}^{2} \approx \frac{\kappa v_{1} v_{2}^{2}}{2 v_{3}}, \quad m_{H_{2}}^{2} \approx 2 \lambda_{1} v_{2}^{2}$.

Observe that, for the hierarchy of the VEVs assumed here, we have that $\mathrm{H}_{2}$ will play the role of the standard Higgs, while $\mathrm{H}_{3}$ is a heavy Higgs, with mass at around the $\mathrm{TeV}$ scale, and $H_{1}$ is a light one with mass at the $\mathrm{eV}$ scale.

In the $C P$-odd sector, things are much simpler, and the mass matrix in the basis $\left(I_{1}, I_{2}, I_{3}\right)$ is given by

$$
M_{I}^{2}=\left(\begin{array}{ccc}
\frac{1}{2} \kappa v_{2}^{2} \frac{v_{3}}{v_{1}} & \kappa v_{2} v_{3} & \frac{1}{2} \kappa v_{2}^{2} \\
\kappa v_{2} v_{3} & 2 \kappa v_{1} v_{3} & \kappa v_{1} v_{2} \\
\frac{1}{2} \kappa v_{2}^{2} & \kappa v_{1} v_{2} & \frac{1}{2} \kappa v_{2}^{2} \frac{v_{1}}{v_{3}}
\end{array}\right) \text {. }
$$

Its diagonalization leads to a Goldstone boson, $G$, that is dominantly $I_{2}$ and will be eaten by the standard gauge boson $Z$, a massless pseudoscalar, $J$, which we call the Majoron, and a heavy pseudoscalar, $A$, which is dominantly $I_{3}$. The relation among these pseudoscalars with the basis is given by

$$
\left(\begin{array}{c}
J \\
G \\
A
\end{array}\right)=U_{I}\left(\begin{array}{c}
I_{1} \\
I_{2} \\
I_{3}
\end{array}\right)
$$

where $U_{I}$ is given by

$$
U_{I} \approx\left(\begin{array}{ccc}
1 & -2 \frac{v_{3}^{2}}{v_{1} v_{2}} & -\epsilon \\
0 & 1 & -2 \frac{v_{3}}{v_{2}} \\
\epsilon & 2 \frac{v_{3}}{v_{2}} & 1
\end{array}\right) .
$$

For the case of interest here, the Majoron is related to the basis in the following way,

$$
J \approx I_{1}-2 \frac{v_{3}^{2}}{v_{2} v_{1}} I_{2}-\epsilon I_{3},
$$

which allows us to conclude that it is dominantly singlet. In this case, its coupling to the standard neutrinos may be approximated by $\sim i g_{\alpha \beta} \bar{\nu}_{\alpha} \gamma_{5} \nu_{\beta} J$, where $g_{\alpha \beta}=Y_{L_{\alpha \beta}} \epsilon$. For $\epsilon=10^{-5}$ and for the values of $Y_{L_{\alpha \beta}}$ given in Eq. (14), we have $g_{e \mu} \sim 10^{-8}$. This value for $g_{e \mu}$ is in agreement with supernova bounds, $\beta \beta 0 \nu$ decay, and neutrino decays, as discussed in [11]. Another source of constraint on the Majoron arises from star cooling, which, for our hierarchy of VEVs, puts the following constraint over them [see Eq. (36) of the first paper in Ref. [5] ]:

$$
\frac{2 v_{3}^{2}}{v_{1} v_{2}} \leq 10^{-6}
$$

which is completely obeyed by our choice in Eq. (12).

Regarding long-range force, the Majoron is so weakly coupled to matter that it may well have escaped detection [12].

The mass of the pseudoscalar $A$ takes the following expression,

$$
m_{A}^{2} \approx \frac{\kappa v_{1} v_{2}^{2}}{2 v_{3}},
$$

which allows us to conclude that it is a heavy particle even for the set of VEVs considered here.

Regarding the charged scalars, in considering the basis $\left(\Delta^{+}, \phi^{+}\right)$, we have the following mass matrix for these scalars:

$M_{H^{ \pm}}^{2}=\left(\begin{array}{cc}\kappa v_{1} v_{3}-\frac{1}{2} \lambda_{5} v_{3}^{2} & \frac{1}{2 \sqrt{2}} v_{2}\left(\lambda_{5} v_{3}-2 \kappa v_{1}\right) \\ \frac{1}{2 \sqrt{2}} v_{2}\left(\lambda_{5} v_{3}-2 \kappa v_{1}\right) & \frac{1}{4 v_{3}} v_{2}^{2}\left(2 \kappa v_{1}-\lambda_{5} v_{3}\right)\end{array}\right)$.

We can easily diagonalize this matrix and find the physical fields

$$
\begin{gathered}
\left(\begin{array}{c}
G^{ \pm} \\
H^{ \pm}
\end{array}\right)=U_{ \pm}\left(\begin{array}{c}
\phi^{ \pm} \\
\Delta^{ \pm}
\end{array}\right), \\
U_{ \pm} \approx\left(\begin{array}{cc}
1 & \frac{\sqrt{2} v_{3}}{v_{2}} \\
-\frac{\sqrt{2} v_{3}}{v_{2}} & 1
\end{array}\right) .
\end{gathered}
$$

We see that there is not any relevant mixing between the charged fields. $G^{ \pm}$is the Goldstone while $H^{ \pm}$is 
the simply charged scalar whose mass expression is given by

$$
m_{H^{ \pm}}^{2}=\frac{1}{4 v_{3}}\left(2 \kappa v_{1}-\lambda_{5} v_{3}\right)\left(v_{2}^{2}+2 v_{3}^{2}\right) \approx \frac{\kappa v_{1} v_{2}^{2}}{2 v_{3}} .
$$

Observe that it must be heavy for the choice of the VEVs used here.

The doubly charged scalar acquires the following mass expression,

$$
m_{\Delta^{ \pm}}^{2}=\frac{1}{2 v_{3}}\left(\kappa v_{1} v_{2}^{2}-2 \lambda_{4} v_{3}^{2}-\lambda_{5} v_{2}^{2} v_{3}\right) \approx \frac{\kappa v_{1} v_{2}^{2}}{2 v_{3}},
$$

which must be heavy, too.

Thus, we see have that, although the VEVs $v_{1}$ and $v_{3}$ are much smaller than $v_{2}$, we have that the scalars that belong to the triplet $\Delta$ are heavier than the standardlike Higgs and their masses are practically determined by the parameter $\kappa$. This is a consequence of the hierarchy of the VEVs. It is curious that the same hierarchy among the VEVs does the opposite with regard to the scalars belonging to the singlet $\sigma$. The scenario predicts a light scalar $H_{1}$. The heavy scalars may be probed at the LHC, while the massless $J$ and light $H_{1}$ will contribute to the invisible decay channels of the Higgs and $Z$.

\section{B. Some constraints}

The coupling constants $\kappa, \beta_{2}$, and $\lambda_{3,5}$ will play an important role in the RGE of the quartic coupling of the standardlike Higgs $\lambda_{1}$. Thus, information on these parameters in the form of constraints is mandatory for us to determine whether or not the vacuum of the 3-2-1 model in the regime of low-energy scale is stable. But before we address this issue, let us investigate the contributions of the light scalars to the invisible decay of the standard neutral gauge boson $Z$.

Regarding the invisible decay of $Z$, the Lagrangian of interest is given by

$$
\mathcal{L}_{R_{3} I_{3} Z} \supset-\frac{g}{c_{w}} Z^{\mu}\left[R_{3} \partial_{\mu} I_{3}-I_{3} \partial_{\mu} R_{3}\right] .
$$

Because $R_{3}$ mixes with $R_{1}$ to compose $H_{1}$ and $I_{3}$ mixes with $I_{1}$ to compose $J$, we have that this Lagrangian generates an interaction among $Z, H_{1}$, and $J$ modulated by the following vertex:

$$
V_{Z H_{1}\left(P_{1}\right) J\left(P_{2}\right)} \approx \frac{g \epsilon^{2}}{c_{W}}\left(P_{1}-P_{2}\right)_{\mu}
$$

where $g$ is the $S U(2)$ coupling constant and $c_{W}=\cos \left(\theta_{W}\right)$, with $\theta_{W}$ being the Weinberg angle. $\epsilon$ is given in Eq. (21). The current data give $\Gamma(Z)_{\text {inv }}=500.1 \pm 1.9 \mathrm{MeV}$ [13]. Because $M_{H_{1}} \ll M_{Z}$, the vertex above provides the following expression for the decay width $Z \rightarrow H_{1} J$,
$\Gamma\left(Z \rightarrow J H_{1}\right)=\frac{M_{Z} \epsilon^{4} G_{F}}{16 \sqrt{2} \pi}\left(M_{Z}-\frac{M_{H_{1}}^{2}}{M_{Z}}\right)^{2} \approx \frac{M_{Z}^{3} \epsilon^{4} G_{F}}{16 \sqrt{2} \pi}$.

The expression for the decay width of $Z$ in two neutrinos is given by

$$
\Gamma(Z \rightarrow \bar{\nu} \nu)=\frac{G_{F} M_{Z}^{3}}{12 \sqrt{2} \pi} .
$$

Upon substituting in the current values of the standard parameters entered in the expression above, i.e., $M_{Z}=$ $91.18 \mathrm{GeV}, G_{F}=1.1663787 \times 10^{-5} \mathrm{GeV}^{-2}$, we obtain $\Gamma(Z \rightarrow \bar{\nu} \nu) \approx 166 \mathrm{MeV}$. In view of this, the window for new physics is established by $\Gamma(Z)_{\text {inv }}-3 \times \Gamma(Z \rightarrow \bar{\nu} \nu) \approx$ 2.1 MeV. In other words, all new contributions to the invisible decay of $Z$ must lie within $2.1 \mathrm{MeV}$.

Observe that Eqs. (36) and (37) provide

$$
\frac{\Gamma_{Z \rightarrow J H_{1}}}{\Gamma_{Z \rightarrow \bar{\nu} \nu}} \approx 0.75 \epsilon^{4} \rightarrow \Gamma_{Z \rightarrow J H_{1}} \approx 124.5 \epsilon^{4} \mathrm{MeV}
$$

According to this, we have that $\Gamma_{Z \rightarrow J H_{1}}$ must be smaller than 2.1 MeV. Once $\frac{v_{3}}{v_{1}}=\epsilon$, at the end of the day we get

$$
\epsilon<0.36 \rightarrow v_{1}>2.77 v_{3} .
$$

This result confirms the hierarchy among the VEVs that we are considering here.

To check that our scenario obeys the constraint put by the invisible decay of $Z$ as discussed above, see that for $v_{1}=10^{5} \mathrm{eV}$ and $v_{3}=1 \mathrm{eV}$ we get $\Gamma\left(Z \rightarrow J H_{1}\right)=$ $124.5 \times 10^{-20} \mathrm{MeV}$, which is much smaller than $2.1 \mathrm{MeV}$. The other possible contribution to $\Gamma(Z)_{\text {inv }}$ is $\Gamma\left(Z^{0} \rightarrow\right.$ $J J J)$. However, we must have that $\Gamma\left(Z^{0} \rightarrow J H_{1}\right)>$ $\Gamma(Z \rightarrow J J J)$ because the later decay is obtained from the first by means of the decay $H_{1} \rightarrow J J$. Thus, we conclude here that the invisible $Z$ decay is not a threat to our model.

Now let us extract constraints over the parameters of the potential by means of the invisible Higgs decay channels and the LFV process $\mu \rightarrow e \gamma$.

Let us consider the contributions that our case gives to the invisible decay of the standardlike Higgs $\mathrm{H}_{2}$. We consider the following contributions: $\Gamma\left(H_{2} \rightarrow H_{1} H_{1}\right)$ and $\Gamma\left(H_{2} \rightarrow J J\right)$. Their decay widths take the expression [14]

$$
\begin{aligned}
\Gamma\left(H_{2} \rightarrow H_{1} H_{1}\right) & \approx \frac{\beta_{2}^{2} v_{2}}{128 \sqrt{2} \pi} \text { and } \\
\Gamma\left(H_{2} \rightarrow J J\right) & \approx \frac{\left(\lambda_{3}+\lambda_{5}\right)^{2} v_{2}}{128 \sqrt{2} \pi} .
\end{aligned}
$$

The prediction for the total decay width of the standard Higgs is around $4 \mathrm{MeV}$, with $\sim 20 \%$ being invisible decay rates $\left[\operatorname{BR}\left(H_{2} \rightarrow\right.\right.$ inv $\left.)=0,26 \pm 0,17\right]$. This allows us to 
conclude that $\beta_{2}, \lambda_{3}$, and $\lambda_{5}$ are constrained to lie at around $10^{-2}$ or smaller.

Thus, we conclude here that the 3-2-1 model lies in the regime of the low-energy scale. Although it has a Majoron, which is a massless pseudoscalar, and a light $C P$-even scalar, it is a safe model for the invisible decay of the standard neutral gauge boson $Z$. As a nice fact, we have that our particular case gives reasonable contribution to the invisible decay of the standard Higgs through the channels $\Gamma\left(H_{2} \rightarrow H_{1} H_{1}\right)$ and $\Gamma\left(H_{2} \rightarrow J J\right)$. In other words, our case may be constrained by future improvements of the data relating to Higgs physics.

Regarding LFV processes, the muon decay channel $\mu \rightarrow e \gamma$ provides the strongest constraint on the parameters of the potential. This is so because, in our case, $\operatorname{BR}(\mu \rightarrow 3 e) \sim \frac{\operatorname{BR}(\mu \rightarrow e \gamma)}{160}$; see Eq. (70) of Ref. [15].

In one-loop order, we have the following expression for the branching ratio of this process [16]:

$$
\begin{aligned}
& \operatorname{BR}(\mu \rightarrow \gamma e) \\
& \quad \approx \frac{27 \alpha\left|\left(Y_{L}\right)_{11}\left(Y_{L}\right)_{12}+\left(Y_{L}\right)_{13}\left(Y_{L}\right)_{32}+\left(Y_{L}\right)_{12}\left(Y_{L}\right)_{22}\right|^{2}}{64 \pi G_{F}^{2} M_{\Delta^{++}}^{4}},
\end{aligned}
$$

where $\alpha$ is the fine structure constant and $G_{F}=$ $1.1663787 \times 10^{-5} \mathrm{GeV}^{-2}$.

Upon substituting in the expression of the mass of the doubly charged scalar given in Eq. (33), we have for the fixed values of $Y_{L}$ 's given in Eq. (14) and of the VEVs given in Eq. (12) that the upper bound $\operatorname{BR}(\mu \rightarrow \gamma e)<$ $5.7 \times 10^{-13}$ [17] translates to the following lower bound over $\kappa$ :

$$
\frac{7 \times 10^{-19}}{\kappa^{2}}<5.7 \times 10^{-13} \rightarrow \kappa>1.1 \times 10^{-3} .
$$

In addition to this lower bound over $\kappa$, there is a natural upper bound over $\kappa$, too, that arises from the constraint that all dimensionless parameters in any scalar potential are required to be less than $\sqrt{4 \pi}$ to fulfill the perturbativity condition. In summary, we have

$$
1.1 \times 10^{-1}<\kappa<\sqrt{4 \pi} .
$$

With this set of constraints in hand, we are ready to analyze the RGE of the quartic coupling of the standardlike Higgs $\lambda_{1}$.

\section{VACUUM STABILITY}

Now that we have developed the scalar sector by finding the spectrum of scalars for a particular set of values of the VEVs and obtained some constraints over the parameters of the potential due to Higgs invisible decay and lepton flavor violation, it is time to investigate the stability of the vacuum by finding the bound from below conditions and calculating the running of the self-coupling of the Higgs.

\section{A. Bound from below conditions}

To assure that the scalar potential of the 3-2-1 model is bounded from below at large field strength, where the potential is generically dominated by the quartic terms, we need to find the set of conditions that guarantee that the parameters of the quartic couplings of the potential are positive when the fields go to infinity. We find the whole set of conditions and pave the way for similar models. We follow the techniques employed in [18].

First, we separate the quartic couplings of the potential,

$$
\begin{aligned}
V^{4}= & \lambda_{1}\left(\Phi^{\dagger} \Phi\right)^{2}+\lambda_{2}\left[\operatorname{tr}\left(\Delta^{\dagger} \Delta\right)\right]^{2}+\lambda_{3} \Phi^{\dagger} \Phi \operatorname{tr}\left(\Delta^{\dagger} \Delta\right) \\
& +\lambda_{4} \operatorname{tr}\left(\Delta^{\dagger} \Delta \Delta^{\dagger} \Delta\right)+\lambda_{5}\left(\Phi^{\dagger} \Delta^{\dagger} \Delta \Phi\right) \\
& +\beta_{1}\left(\sigma^{*} \sigma\right)^{2}+\beta_{2} \Phi^{\dagger} \Phi \sigma^{*} \sigma+\beta_{3} \operatorname{tr}\left(\Delta^{\dagger} \Delta\right) \sigma^{*} \sigma \\
& -\kappa\left(\Phi^{T} \Delta \Phi \sigma+\text { H.c. }\right)
\end{aligned}
$$

and we then build the following parametrization:

$$
\begin{aligned}
r^{2} & =\Phi^{\dagger} \Phi+\operatorname{tr}\left(\Delta^{\dagger} \Delta\right)+\sigma^{*} \sigma, \\
\Phi^{\dagger} \Phi & =r^{2} \cos ^{2} \gamma \sin ^{2} \theta, \\
\operatorname{tr}\left(\Delta^{\dagger} \Delta\right) & =r^{2} \sin ^{2} \gamma \sin ^{2} \theta, \\
\sigma^{*} \sigma & =r^{2} \cos ^{2} \theta,
\end{aligned}
$$

where $0 \leq r \leq \infty, 0 \leq \gamma \leq \frac{\pi}{2}$, and $0 \leq \theta \leq \frac{\pi}{2}$.

We also need to develop the following parameters,

$$
\begin{aligned}
\zeta & =\frac{\operatorname{tr}\left(\Delta^{\dagger} \Delta \Delta^{\dagger} \Delta\right)}{\left[\operatorname{tr}\left(\Delta^{\dagger} \Delta\right)\right]^{2}}, \\
\xi & =\frac{\Phi^{\dagger} \Delta \Delta^{\dagger} \Phi}{\Phi^{\dagger} \Phi \operatorname{tr}\left(\Delta^{\dagger} \Delta\right)}, \\
\alpha & =\frac{\operatorname{Re}\left(\Phi^{T} \Delta \Phi \sigma\right)}{\operatorname{tr}\left(\Delta^{\dagger} \Delta\right) \sigma^{*} \sigma+\Phi^{\dagger} \Phi \sigma^{*} \sigma+\operatorname{tr}\left(\Delta^{\dagger} \Delta\right) \Phi^{\dagger} \Phi},
\end{aligned}
$$

where $\frac{1}{2} \leq \zeta \leq 1,0 \leq \xi \leq 1$, and $-1 \leq \alpha \leq 1$. Two of them are already known in the literature. The third one is a new parameter. We can see in detail in the Appendix how we can limit this parameter.

Let us also define new variables $x$ and $y$ that must vary between 0 and 1 in the following way: 


$$
\begin{aligned}
& y=\sin ^{2} \theta, \\
& x=\sin ^{2} \gamma .
\end{aligned}
$$

Replacing Eq. (47) with Eq. (44), we get

$$
\begin{aligned}
\frac{V^{4}}{r^{4}}= & y^{2}\left[\lambda_{1}(1-x)^{2}+\lambda_{2} x^{2}+\lambda_{3}(1-x) x\right. \\
& \left.+\zeta \lambda_{4} x^{2}+\xi \lambda_{5}(1-x) x-2 \kappa \alpha x(1-x)\right] \\
& +(1-y)^{2} \beta_{1}+(1-y) y\left[\beta_{2}(1-x)+\beta_{3} x-2 \kappa \alpha\right] .
\end{aligned}
$$

We manage things such that we can express these quartic terms in the following way:

$$
\begin{aligned}
& \frac{V^{4}}{r^{4}}=A_{x} y^{2}+B_{x}(1-y)^{2}+C_{x}(1-y) y, \text { where } \\
& A_{x}=\lambda_{1}(1-x)^{2}+\left(\lambda_{2}+\zeta \lambda_{4}\right) x^{2}+\left(\lambda_{3}+\xi \lambda_{5}-2 \kappa \alpha\right)(1-x) x, \\
& B_{x}=\beta_{1}, \\
& C_{x}=\beta_{2}(1-x)+\beta_{3} x-2 \kappa \alpha .
\end{aligned}
$$

We can fix $y=0$ or $y=1$ to obtain the cases in which the quartic couplings of the potential is positive. When we do this, we obtain the following conditions:

$$
\begin{gathered}
A_{x}>0, \\
B_{x}>0, \\
C_{x}+2 \sqrt{A_{x} B_{x}}>0 .
\end{gathered}
$$

For $A_{x}>0$, we need to use the same argument as before. Fixing $x=0$ and $x=1$, we have similar conditions for the inequalities,

$$
\begin{aligned}
\lambda_{1} & >0, \\
\lambda_{2}+\zeta \lambda_{4} & >0, \\
\lambda_{3}+\xi \lambda_{5}-2 \kappa \alpha+2 \sqrt{\lambda_{1}\left(\lambda_{2}+\zeta \lambda_{4}\right)} & >0 .
\end{aligned}
$$

These new conditions depend of the parameters in Eq. (49). They vary in different ranges, but we only need to study the boundary values of these intervals. In this case, the new conditions are

$$
\begin{aligned}
\lambda_{1} & >0, \\
\lambda_{2}+\lambda_{4} & >0, \\
\lambda_{2}+\frac{1}{2} \lambda_{4} & >0, \\
\lambda_{3}+2 \kappa+2 \sqrt{\lambda_{1}\left(\lambda_{2}+\frac{1}{2} \lambda_{4}\right)} & >0, \\
\lambda_{3}+2 \kappa+2 \sqrt{\lambda_{1}\left(\lambda_{2}+\lambda_{4}\right)} & >0, \\
\lambda_{3}+\lambda_{5}+2 \kappa+2 \sqrt{\lambda_{1}\left(\lambda_{2}+\frac{1}{2} \lambda_{4}\right)} & >0, \\
\lambda_{3}+\lambda_{5}+2 \kappa+2 \sqrt{\lambda_{1}\left(\lambda_{2}+\lambda_{4}\right)} & >0, \\
\lambda_{3}-2 \kappa+2 \sqrt{\lambda_{1}\left(\lambda_{2}+\frac{1}{2} \lambda_{4}\right)} & >0, \\
\lambda_{3}-2 \kappa+2 \sqrt{\lambda_{1}\left(\lambda_{2}+\lambda_{4}\right)} & >0, \\
\lambda_{3}+\lambda_{5}-2 \kappa+2 \sqrt{\lambda_{1}\left(\lambda_{2}+\frac{1}{2} \lambda_{4}\right)} & >0, \\
\lambda_{3}+\lambda_{5}-2 \kappa+2 \sqrt{\lambda_{1}\left(\lambda_{2}+\lambda_{4}\right)} & >0 .
\end{aligned}
$$

Using the same argument as for the condition in Eq. (51), it is easy to see that

$$
\beta_{1}>0
$$

Using the condition in Eq. (52) and the same fact that $C_{x}$ can have $x=0$ or $x=1$, we obtain

$$
\begin{aligned}
\beta_{2}-2 \kappa \alpha+2 \sqrt{\beta_{1} \lambda_{1}} & >0, \\
\beta_{3}-2 \kappa \alpha+2 \sqrt{\left.\beta_{1}\left(\lambda_{2}+\zeta \lambda_{4}\right)\right)} & >0 .
\end{aligned}
$$

The first inequality has two different solutions, while the second has four. At the end of the day, we have

$$
\begin{aligned}
\beta_{2}+2 \kappa+2 \sqrt{\beta_{1} \lambda_{1}} & >0, \\
\beta_{2}-2 \kappa+2 \sqrt{\beta_{1} \lambda_{1}} & >0, \\
\beta_{3}+2 \kappa+2 \sqrt{\beta_{1}\left(\lambda_{2}+\frac{1}{2} \lambda_{4}\right)} & >0, \\
\beta_{3}+2 \kappa+2 \sqrt{\beta_{1}\left(\lambda_{2}+\lambda_{4}\right)} & >0, \\
\beta_{3}-2 \kappa+2 \sqrt{\beta_{1}\left(\lambda_{2}+\frac{1}{2} \lambda_{4}\right)} & >0, \\
\beta_{3}-2 \kappa+2 \sqrt{\beta_{1}\left(\lambda_{2}+\lambda_{4}\right)} & >0 .
\end{aligned}
$$

So, that is the set of conditions that guarantees that the potential in Eq. (3) is bounded from below. In what follows, 

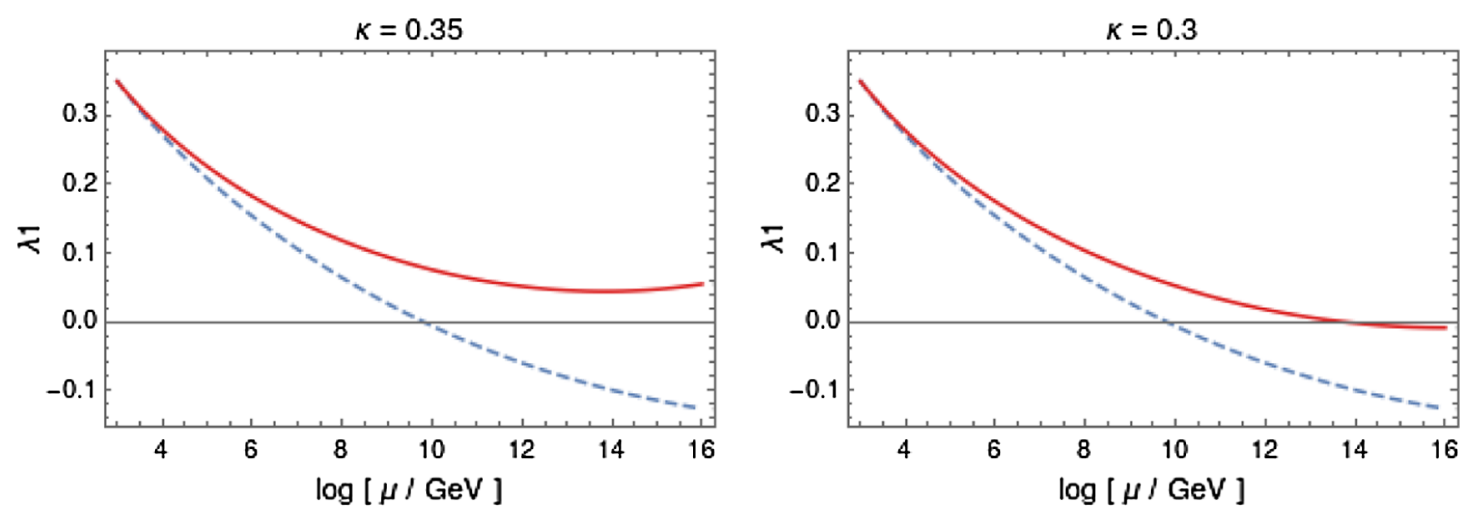

FIG. 1. Running of $\lambda_{\Phi} \approx \lambda_{1}$ at one-loop level as a function of the energy scale $\mu$ for $\lambda_{5}=\lambda_{3}=\beta_{2}=0.001$ with $y_{t}=0.9965$, $g_{Y}=0.4627$, and $g=0.6535$. The dashed lines represent the expectation of the standard model, and the red lines represent the expectation for our model for two values of $\kappa$.

we obtain the running of the self-coupling related to the standardlike Higgs.

\section{B. RGE of the self-coupling of the standardlike Higgs}

The standard model predicts that the self-coupling of the Higgs becomes negative at an energy scale of around $\Lambda=10^{11} \mathrm{GeV}$. This means that the standard model cannot assure the stability of the vacuum up to the Planck scale. This must be remedied by means of new physics in the form of new particles with appropriate interactions. This issue has been extensively investigated in the literature [19]. In our scenario, we show that the right behavior of the selfcoupling of the Higgs that guarantees absolute stability for the electroweak vacuum depends strongly on the coupling $\kappa$. We do our analysis by implementing the model in SARAH4.13.0 [20] and evaluating the $\beta$ function for $\lambda_{1}$ at one-loop level.

The main contributions for the beta function of $\lambda_{1}$ involve the following terms:

$$
\begin{aligned}
\beta_{\lambda_{1}}= & \frac{27}{100} g_{Y}^{4}+\frac{9}{4} g^{4}+\frac{9}{20} g_{Y}^{2}\left(g^{2}-2 \lambda_{1}\right)-\frac{9}{5} g^{2} \lambda_{1}+12 \lambda_{1}^{2} \\
& +12 \lambda_{1} y_{t}^{\dagger} y_{t}-6 y_{t}^{\dagger} y_{t} y_{t}^{\dagger} y_{t} \\
& +\beta_{2}^{2}+3 \lambda_{3}^{2}+3 \lambda_{3} \lambda_{5}+\frac{5}{4} \lambda_{5}^{2}+2 \kappa^{2},
\end{aligned}
$$

where $g$ and $g_{Y}$ are the gauge couplings of the standard gauge group $S U(2)$ and $U(1)_{Y}$, while $y_{t}$ is the Yukawa coupling of the quark top.

Observe that the couplings $\beta_{2}, \lambda_{3,5}$, and $\kappa$ give positive contributions to the running of $\lambda_{1}$. However, as showed above, the invisible Higgs decay requires $\beta_{2}, \lambda_{3,5}$ less than $10^{-2}$, which turns insignificant their contributions to the RGE. We put our hope on the contribution of the parameter $\kappa$. In Fig. 1, we show the plot of the running of $\lambda_{1}$ with an energy scale for two possible values of $\kappa$. We see that the running of $\lambda_{1}$ may get positive up to Planck scale for $\kappa>0.3$. Thus, the model may have the vacuum remain stable thanks to the contribution of the parameter $\kappa$.

For the sake of completeness, in Figs. 2 and 3 we present the running at one-loop level of the other self-quartic

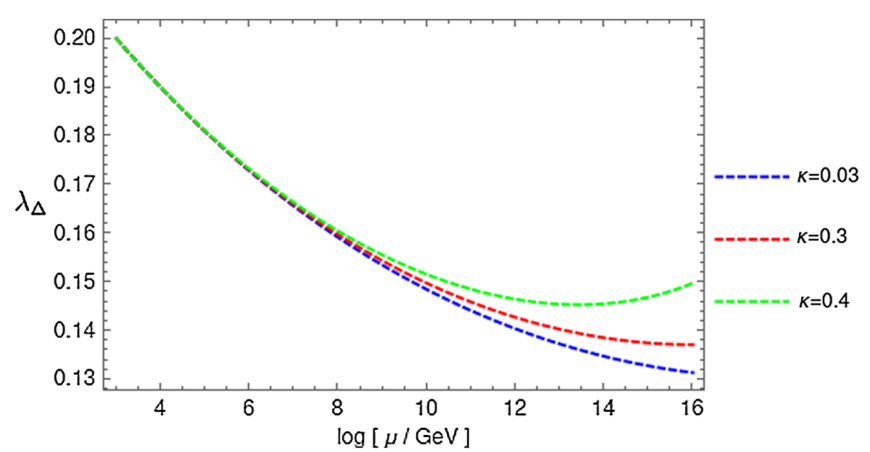

FIG. 2. Running of $\lambda_{\Delta} \approx \lambda_{2}+\lambda_{4}$ at one-loop level as a function of the energy scale $\mu$ for $\lambda_{5}=\lambda_{3}=\beta_{2}=0.001$ with $y_{t}=0.9965$, $g_{Y}=0.4627$, and $g=0.6535$. The dashed lines represent the expectation for our model for three values of $\kappa$. The coupling $\beta_{3}$ is important at the same level as $\kappa$ as for this running.

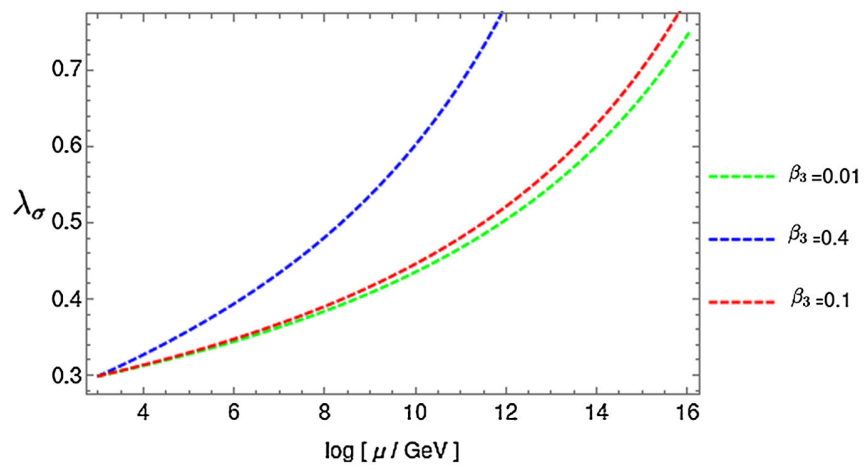

FIG. 3. Running of $\lambda_{\sigma} \approx \beta_{1}$ at one-loop level as a function of the energy scale $\mu$ for $\lambda_{5}=\lambda_{3}=\beta_{2}=0.001$ with $y_{t}=0.9965$, $g_{Y}=0.4627$, and $g=0.6535$. The dashed lines represent the expectation for our model for three values of $\beta_{3}$. 
couplings $\lambda_{2,4}$ and $\beta_{1}$. As we can see in those plots, they do not develop negative values and are weakly influenced by $\kappa$.

\section{CONCLUDING REMARKS}

In this work, we studied stability of the vacuum in the 3-2-1 model with right-handed neutrinos. We restricted our investigation to a specific case where lepton number is spontaneously broken at low-energy scale. The scenario is well motivated since it yields light sterile neutrinos and may explain MiniBooNE results by means of neutrino oscillation. In such a scenario, we obtained the whole set of conditions that guarantee that the model is bounded from below and studied the RGE of the self-coupling of the standardlike Higgs. For our main result, we have that the quartic coupling $\kappa \Phi^{T} \Delta \Phi \sigma$ plays a central role in the process and that the stability of the vacuum requires $\kappa>0.3$.

As an interesting consequence, we remark that the model has one Majoron $(J)$ and one light Higgs $\left(H_{1}\right)$ composing the spectrum of scalar of the model. Their contributions to the invisible decay rate of the standardlike Higgs, $\mathrm{H}_{2} \rightarrow J J$ and $H_{2} \rightarrow H_{1} H_{1}$, were considered and the results are the bounds $\beta_{2}, \lambda_{3}, \lambda_{5} \leq 10^{-2}$ over the couplings of the potential.

Regarding the neutrino sector, the scenario recovers the $3+1$ sterile neutrino model which explain the MiniBooNE experiment by means of neutrino oscillation. However, we know that light sterile neutrinos are strongly disfavored by current cosmological data involving big bang nucleosynthesis, cosmic microwave background anisotropies, and large scale structure [10]. This is so because, in the face of the large mixing required by MiniBooNE, neutrino oscillation may conduct sterile neutrino to thermal equilibrium with the active neutrino even before neutrinos decouple from the primordial plasma. A possible solution for this tension requires the suppression of the production of these neutrinos in the early Universe. This keeps them from thermalizing with the active ones at high temperature. This may be achieved by means of secret interactions [21], which is nothing more than the interaction of the sterile neutrino with a pseudoscalar, $I$,

$$
\sim g_{s} \bar{\nu}_{S}^{C} \gamma_{5} \nu_{S} I \text {. }
$$

The solution to the tension requires $I$ to be lighter than the lightest sterile neutrino and $g_{s}$ to take values in the range $10^{-6}-10^{-5}$. Observe that our scenario recovers this solution. For this, recognize that $g$ is $Y_{11}^{R}$, whose value in the matrix in Eq. (15) is $1,4 \times 10^{-5}$, and $I$ is the Majoron $J$. To generate a small mass for $J$, we just need to consider a term like $M \sigma \sigma \sigma$ in the potential. This term will generate a mass term for $J$ proportional to $M$. Assuming that $M<m_{N_{4}}$, we have a secret sector that reconciliates an $\mathrm{eV}$ sterile neutrino with cosmology as was done in [22].

\section{ACKNOWLEDGMENTS}

J. P. P thanks CNPq for the financial support. C. A. S. P. was supported by CNPq Research Grant No. 304423/ 2017-3.

\section{APPENDIX}

Here we will give a hint of the proof of the limitation of the parameter $\alpha$. The definition of this parameter is

$$
\alpha=\frac{\operatorname{Re}\left[\Phi^{T} \Delta \Phi \sigma\right]}{\Phi^{\dagger} \Phi \sigma^{\dagger} \sigma+\operatorname{tr}\left[\Delta^{\dagger} \Delta\right] \Phi^{\dagger} \Phi+\operatorname{tr}\left[\Delta^{\dagger} \Delta\right] \sigma^{\dagger} \sigma} .
$$

We can expand this parameter in terms of the components of the fields. We have that

$$
\begin{aligned}
\text { Numerator }= & \operatorname{Re}\left[\phi^{0} \Delta^{0} \phi^{0} \sigma+\sqrt{2} \phi^{0} \Delta^{+} \phi^{-} \sigma\right. \\
& \left.+\phi^{-} \Delta^{++} \phi^{-} \sigma\right], \\
\text { Denominator }= & \left(\phi^{0 \dagger} \phi^{0}+\phi^{+} \phi^{-}+\sigma^{\dagger} \sigma\right) \\
& \times\left(\Delta^{0 \dagger} \Delta^{0}+\Delta^{+} \Delta^{-}+\Delta^{++} \Delta^{--}\right) \\
& +\sigma^{\dagger} \sigma\left(\phi^{0 \dagger} \phi^{0}+\phi^{+} \phi^{-}\right) .
\end{aligned}
$$

Then we can study term by term to see what is the behavior of this parameter, e.g., to see whether or not it is limited. As an example, we choose the first term of the numerator and expand the fields in the real and imaginary parts. Using the following expansion,

$$
\begin{aligned}
\phi^{0} & =R_{2}+i I_{2}, \\
\Delta^{0} & =R_{3}+i I_{3}, \\
\sigma & =R_{1}+i I_{1},
\end{aligned}
$$

we obtain the denominator terms (only the real part)

$$
\begin{aligned}
& R_{2}^{2} R_{3} R_{1}-R_{2} R_{3} I_{2} I_{1}-I_{2} I_{3} R_{2} R_{1}+I_{1} I_{2}^{2} I_{3}-I_{2}^{2} R_{1} R_{3} \\
& \quad-I_{1} I_{2} R_{2} R_{3}-R_{1} R_{2} R_{2} I_{3}-R_{2}^{2} I_{1} I_{3} .
\end{aligned}
$$

The idea here is to look closely at each real function and study their limitation range. For the first term, $R_{2}^{2} R_{3} R_{1}$, we have the following relation (for $R_{2} \neq 0$ ):

$$
\begin{aligned}
\frac{R_{2}^{2} R_{3} R_{1}}{R_{2}^{2} R_{3}^{2}+R_{1}^{2} R_{2}^{2}+R_{1}^{2} R_{3}^{2}+(\ldots)} & \rightarrow \frac{R_{3} R_{1}}{R_{3}^{2}+R_{1}^{2}+\frac{R_{1}^{2} R_{3}^{2}}{R_{2}^{2}}+(\ldots)} \\
& <\frac{R_{3} R_{1}}{R_{3}^{2}+R_{1}^{2}} .
\end{aligned}
$$

We can see easily that this last term is limited in the range $[-1,1]$ with polar coordinates. We use similar arguments for the next terms and find that $\alpha$ lies in the range $[-1,1]$. 
[1] G. C. Branco, P. M. Ferreira, L. Lavoura, M. N. Rebelo, M. Sher, and J. P. Silva, Phys. Rep. 516, 1 (2012).

[2] T. P. Cheng and L.-F. Li, Phys. Rev. Lett. 45, 1908 (1980); G. B. Gelmini and M. Roncadelli, Phys. Lett. B 99, 411 (1981).

[3] N. V. Krasnikov, Phys. Lett. B 291, 89 (1992); A. S. Joshipura and J.W.F.Valle, Nucl. Phys. B397, 105 (1993); D. O’Connell, M. J. Ramsey-Musolf, and M. B. Wise, Phys. Rev. D 75, 037701 (2007).

[4] J. Schechter and J. W. E Valle, Phys. Rev. D 25, 774 (1982).

[5] M. A. Diaz, M. A. Garcia-Jareno, D. A. Restrepo, and J. W. F. Valle, Nucl. Phys. B527, 44 (1998); C. Bonilla, J. C. Romo, and J. W. F. Valle, New J. Phys. 18, 033033 (2016); S. Blunier, G. Cottin, M. Aurelio Daz, and B. Koch, Phys. Rev. D 95, 075038 (2017).

[6] For an extension of this scenario involving the two-Higgsdoublet model, see D. Cogollo, R. D. Matheus, T. B. de Melo, and F.S. Queiroz, Phys. Lett. B 797, 134813 (2019).

[7] A. Aguilar-Arevalo et al., Phys. Rev. Lett. 121, 221801 (2018).

[8] M. J. G. Veltman, Nucl. Phys. B123, 89 (1977).

[9] A. Aguilar-Arevalo et al., Phys. Rev. D 64, 112007 (2001).

[10] G. Steigman, Adv. High Energy Phys. 2012, 268321 (2012); N. Aghanim et al. (Planck Collaboration), arXiv:1807 .06209; J. Hamann, S. Hannestad, G. G. Raffelt, and Y. Y. Y. Wong, J. Cosmol. Astropart. Phys. 09 (2011) 034.

[11] A. P. Lessa and O. L. G. Peres, Phys. Rev. D 75, 094001 (2007).

[12] Y. Chikashige, R. N. Mohapatra, and R. D. Peccei, Phys. Lett. 98B, 265 (1981); F. Ferrer and M. Nowakowski, Phys. Rev. D 59, 075009 (1999).

[13] M. Tanabashi et al. (Particle Data Group), Phys. Rev. D 98, 030001 (2018) and 2019 update.
[14] C. Bonilla, J. C. Romo, and J. W. F. Valle, New J. Phys. 18, 033033 (2016).

[15] Y. Kuno and Y. Okada, Rev. Mod. Phys. 73, 151 (2001).

[16] A. G. Akeroyd, M. Aoki, and H. Sugiyama, Phys. Rev. D 79, 113010 (2009); For a general formula for $f_{1} \rightarrow f_{2} \gamma$, see L. Lavoura, Eur. Phys. J. C 29, 191 (2003).

[17] MEG Collaboration, Phys. Rev. Lett. 110, 201801 (2013).

[18] A. Arhrib, R. Benbrik, M. El Kacimi, L. Rahili, and S. Semlali, Eur. Phys. J. C 80, 13 (2020); D. A. Camargo, A. G. Dias, T. B. de Melo, and F. S. Queiroz, J. High Energy Phys. 04 (2019) 129; G. Chauhan, J. High Energy Phys. 12 (2019) 137.

[19] I. Gogoladze, N. Okada, and Q. Shafi, Phys. Rev. D 78, 085005 (2008); B. He, N. Okada, and Q. Shafi, Phys. Lett. B 109, 197202 (2012); J. Chakrabortty, M. Das, and S. Mohanty, Mod. Phys. Lett. A 28, 1350032 (2013); A. Kobakhidze and A. Spencer-Smith, J. High Energy Phys. 08 (2013) 036; J. N. Ng and A. de la Puente, Eur. Phys. J. C 76, 122 (2016); I. Garg, S. Goswami, K. N. Vishnudath, and N. Khan, Phys. Rev. D 96, 055020 (2017); S. Ipek, A. D. Plascencia, and J. Turner, J. High Energy Phys. 12 (2018) 111; C. Bonilla, R. M. Fonseca, and J. W. F. Valle, Phys. Rev. D 92, 075028 (2015).

[20] F. Staub, Comput. Phys. Commun. 185, 1773 (2014).

[21] S. Hannestad, R. S. Hansen, and T. Tram, Phys. Rev. Lett. 112, 031802 (2014); M. Archidiacono, S. Gariazzo, C. Giunti, S. Hannestad, R. Hansen, M. Laveder, and T. Tram, J. Cosmol. Astropart. Phys. 08 (2016) 067; B. Dasgupta and J. Kopp, Phys. Rev. Lett. 112, 031803 (2014); X. Chu, B. Dasgupta, M. Dentler, J. Kopp, and N. Saviano, J. Cosmol. Astropart. Phys. 11 (2018) 049.

[22] C. A. de S. Pires, Phys. Lett. B 800, 135135 (2020); D. Croon, N. Fernandez, D. McKeen, and G. White, J. High Energy Phys. 06 (2019) 098. 